\title{
Screening for Birth Defects Strategies for Developing Low Resource Countries
}

Prashant Acharya, Jaideep Malhotra, Narendra Malhotra, S Suresh, Ashok Khurana, Chander Lulla, Hriday Acharya, Neharika Malhotra Bora, Rishabh Bora, Keshav Malhotra

\section{ABSTRACT}

Most low resource countries have no definite policies laid down for screening for fetal abnormalities and prenatal diagnostic techniques. The problem with screening scans and prenatal diagnostic techniques is the variable way in which they are conducted. There are no clear guidelines about what should, or what should not be done. What is needed is a standard for a routine anomaly scan.

In the past 10 to 15 years, major advances have been made in prenatal screening. It has been suggested that maternal age alone as a screening strategy should be abandoned, but there is still no consensus on the most cost-effective alternative, and thus no national strategy exists.

This document will provide parameters for obstetricians, radiologists and sonographers-how much screening could be accomplished within the available resources. With the help of prenatal diagnostic technique guideline we will be able to achieve a methodical, uniform and cost-effective way of fetal evaluation.

Keywords: Birth defects, Low resource, NT, Anomaly scan, Prenatal diagnostic technique, Amniocentesis, Chorion villus sampling, Aneuploidy, Nasal bone, Genetic sonogram.

How to cite this article: Acharya P, Malhotra J, Malhotra N, Suresh S, Khurana A, Lulla C, Acharya H, Bora NM, Bora R, Malhotra K. Screening for Birth Defects Strategies for Developing Low Resource Countries. Donald School J Ultrasound O bstet Gynecol 2013;7(4):453-461.

\section{Source of support $\mathrm{Nil}$}

Conflict of interest: None declared

\section{INTRODUCTION}

In India at present there is no definite policy laid down for the ultrasound screening for fetal abnormalities and the prenatal diagnostic techniques. The problem with screening scans and prenatal diagnostic techniques is the variable way in which they are conducted throughout the country. There are no clear guidelines about what should, or should not, be done. The present working group recommends a national standard for a routine ultrasound anomaly scan. This document will provide parameters for obstetricians, radiologists and sonographers how much screening could be accomplished within the available resources.

With the help of prenatal diagnostic technique guideline, we will be able to achieve methodical, uniform, cost-effective and better fetal evaluation. We are also aiming at lowering the prenatal invasive diagnostic procedures because of the introduction of effectivescreening methods.

\section{Training and Registration}

As per the law in India today, those who fulfill the criteria according to PCPNDT act, can perform the scan. He/she should be sufficiently trained to do sonography and must be registered with appropriate authority.

\section{TO $13+6$ WEEKS SCAN}

The first scan in early pregnancy should be undertaken ideally between $11-13+6$ weeks. The purpose of this scan is to establish:

- Gestational age accurately

- Viability

- Fetal number, and in multiple pregnancies the chorionicity/amnionicity

- Detection of gross fetal abnormalities.

Before 13 weeks, gestational page can be accurately assessed from the measurement of crown rump length $(C R L) .{ }^{2}$ However, from 14 th weeks $C R L$ should not be used because the fetus becomes increasingly flexed making the measurement unreliable. A s an al ternative to $C R L$, bi parietal diameter, and/or head circumference should be used. The early scan can usually be performed by transabdominal and/ or transvaginal route.

\section{TO $13+6$ WEEKS ANEUPLOIDY SCAN}

Correct measurement of $C R L$, nuchal translucency (NT), heart rate must be done. NT is measured when CRL is between 45 and 84 only. Fetal anomaly scan to detect the gross fetal anomaly should be performed. A dditional study of nasal bone (NB), ductus venosus and tricuspid regurgitation can improve the detection rate of fetal aneuploidy. Study of uterine artery Doppler and cervical length can be optional. Reporting of 11-, 13+6 weeks scan must include the risk assessment of trisomy 21 . Consultant who is performing NT scan must be properly trained, preferably certified by Fetal M edicine Foundation, U nited $\mathrm{K}$ ingdom (FM F, UK). The software for calculating the risk of trisomy 21 is available free for those accredited by FM F in 11-13+6 weeks scan. Regular audit report ensures continuity of license for the use of software.

All women have chance to deliver the trisomy 21 baby which is known as 'risk a priori' ${ }^{7} \mathrm{~A}$ s the age increases, risk for delivering a trisomy 21 increases as shown in A ppendix 1. A s far as scanning for NT is concerned, current 
Prashant Acharya et al

\begin{tabular}{lcccc}
\hline \multicolumn{5}{c}{ Appendix 1: Maternal age risk 'a priori' } \\
\hline Maternal & \multicolumn{4}{c}{ Trisomy 21 } \\
\cline { 2 - 5 } age & 12 weeks & 16 weeks & 20 weeks & 40 weeks \\
\hline 20 & 1068 & 1200 & 1295 & 1527 \\
25 & 946 & 1066 & 1147 & 1352 \\
30 & 626 & 703 & 759 & 895 \\
31 & 543 & 610 & 658 & 776 \\
32 & 461 & 518 & 559 & 659 \\
33 & 383 & 430 & 464 & 547 \\
34 & 312 & 350 & 378 & 446 \\
35 & 249 & 280 & 302 & 356 \\
36 & 196 & 220 & 232 & 280 \\
37 & 152 & 171 & 185 & 218 \\
38 & 117 & 131 & 142 & 167 \\
39 & 89 & 100 & 108 & 128 \\
40 & 68 & 76 & 82 & 97 \\
41 & 51 & 57 & 62 & 73 \\
42 & 38 & 42 & 46 & 55 \\
\hline
\end{tabular}

evidence suggests that this is an effective way of determining babies at risk of Down syndrome and is best performed between $11-13+6$ weeks (detection rate $70-80 \%$ with fal sepositive rate of $5 \%$ ). A dding NB to NT improves the detection rate by $10 \%$ (detection rate $90 \%$ with false-positive rate of $5 \%)^{11}$

Adding the serum biochemistry [free beta-human chorionic gonadotropin (hCG) and pregnancy-associated plasma protein A (PAPP-A)] to NT improves the detection rate by $10 \%$ (detection rate $90 \%$ with false-positive rate of $5 \%)$. M aternal serum screening can be used to modify a woman's age-related risks. If serum testing is to be used as a method of screening for Down syndrome, accurate knowledge of gestational age is essential. ${ }^{3,9,11}$ In developing countries where appropriate and authentic laboratory facility for dual or Q uadruple marker tests are NOT available, one should rely on NT, Nasal bone and ductus venosus by appropriately trained person for aneuploidy screening.

\section{Comparison of the Detection Rates, for a False-positive Rate of 5\%, of Different Methods of Screening for Trisomy 21 (www.fetalmedicine.com) $)^{8,11}$}

Combination of age, NT, NB, ductus venosus, tricuspid regurgitation, heart rate with serum biochemistry will produce the highest sensitivity with the lowest fal se positive rate (Table 1$){ }^{1}$

\section{TO 13+ 6 WEEKS ANOMALY SCAN}

Nicolaides et $\mathrm{al}^{23}$ mentioned that almost $43.6 \%$ of fetal anomalies were detected at $11-13+6$ weeks. The $11-13+6$ weeks scan detected all cases of acrania, alobar holoprosencephaly, exomphalos, gastroschisis, megacystis and body stalk anomaly, $77 \%$ of absent hand or foot, $50 \%$

of diaphragmatic hernia, $50 \%$ of lethal skeletal dysplasias, $60 \%$ of polydactyly, $34 \%$ of major cardiac defects, $5 \%$ of facial clefts and $14 \%$ of open spina bifida, but none of agenesis of the corpus callosum, cerebellar or vermian hypoplasia, echogenic lung lesions, bowel obstruction, most renal defects or talipes. NT was above the 95th percentile in $34 \%$ of fetuses with major cardiac defects. Data from various studies by Whithlow et al, Radhakrishnan et al, Suseela et al ${ }^{21,24,25}$ suggest that about from 22,55 and $85 \%$ of significant abnormalities will be identified by a screening scan early in the 11-13+6 weeks scan respectively. These data proves that $11-13+6$ weeks scan is very helpful in the diagnosis of significant lethal fetal anomalies, which are not compatible with life during in the first trimester of pregnancy.

\section{'18 TO 20 WEEKS'-TARGETED ANOMALY SCAN}

When 1st trimester scan is missed, the ' 18 to 20 weeks' targeted anomaly scan provides dating information and diagnosis of multiple pregnancy. The majority of nonviable pregnancies will be lost before the scan at 20 weeks. A $n$ attempt must be made to evaluate the fetus completely with standard views to exclude major structural anomalies in transverse, sagittal and coronal plains. The ' 18 to 20 weeks' targeted anomaly scan is to reassure the woman that the fetus appears to have no obvious structural abnormalities. The primary aim should be to prove the 'normality'. Recent data ${ }^{4}$ from one unit suggested that about $50 \%$ of significant abnormalities would be identified by a screening scan. Recent data from Radhakrishnan et $\mathrm{al}^{21}$ suggested that about $55 \%$ of significant abnormalities will be identified by a screening scan early in the $11-13+6$ weeks scan and detection will go to $88 \%$ after 18 to 20 weeks scan in the expert hands. The ' 18 to 20 weeks' -anomaly scan should 
Screening for Birth Defects Strategies for Developing Low Resource Countries

be carried out at the clinic with minimum standard described below. If a clinic considers that it cannot deliver scans to this minimum standard as described below then the ' 18 to 20 weeks' scan should be referred to an appropriate unit.

\section{Procedure}

The minimum standard for an '18 to 20 weeks' targeted anomaly scan, gestational age can be established by measurement of biparietal diameter, head circumference, abdominal circumference and femur length.

\section{Fetal Normality}

- Head size, shape + internal structures cavum pellucidum cerebellum ventricular size at atrium/parieto-occipital sulcus

- Face, lips with face profile to show nasal bone

- Spine: longitudinal, sagittal and transverse

- Thorax at level of 4-chamber cardiac view and three vessel views

- A bdominal shape and content at level of stomach

- Renal pelvis measurement

- Abdominal shape and content at level of kidneys and umbilicus

- Arms: three bones and hand (not counting fingers)

- Legs: three bones and foot (not counting toes).

If resources are available uterine artery Doppler and the measurement of cervical length should be included in the extended scan. A dding these parameters will improve in identifying the patients at risk of preterm delivery and the consequences of improper placentation, respectively. Fetal heart examination should be carried out in detail with suspected/confirm other fetal anomalies and in all medical situation where the incidence of congenital heart diseases is increased.

A checklist for the baseline fetal anomaly scan is included as Appendix 2. Detection rate of major fetal anomaly is included in A ppendix 3. The risk of aneuploidy should be mentioned when scan is performed between 11 and $13+6$ weeks scan with the help of software. The risk of aneuploidy should be mentioned when scan is performed between 16 and 20 weeks with the help of software or can be calculated with the help of likelihood ratio $(L R)$ for all the soft markers. U se of Indian fetal biometry and growth charts should be encouraged for better prediction of fetal age and the fetal growth for Indian population. A charya et $\mathrm{al}^{10}$ showed the distinct advantages of using the Indian fetal biometry over the western fetal biometry for Indian population. Like wise, use of Biometry and growth charts derived from local race and population will be more helpful in identifying the appropriate fetal growth with better
Appendix 2: Ultrasound screening for aneuploidy and baseline fetal anomaly scan guidance
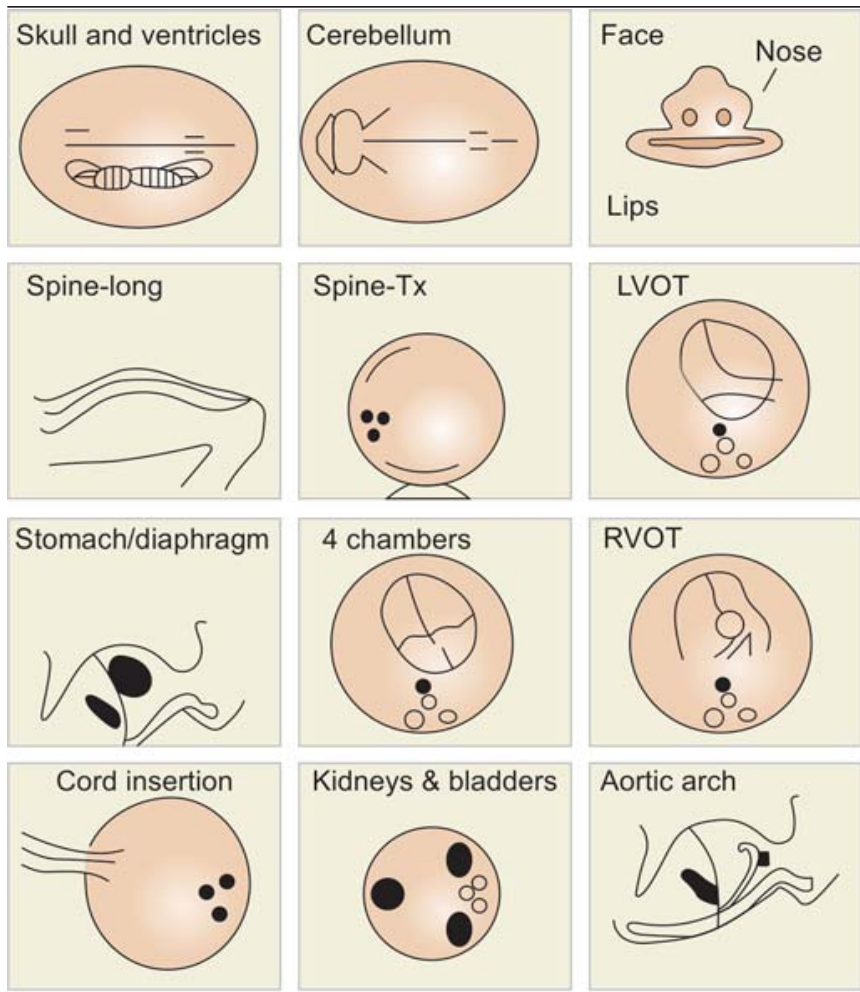

Kidneys \& bladders
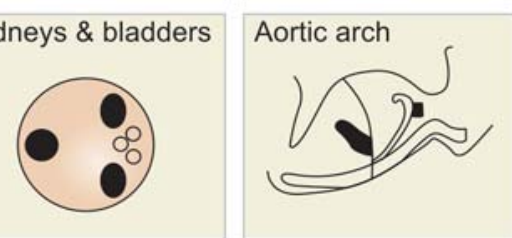

Arms-3 long bones

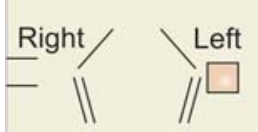

Legs-3 long bones

Orientation of feet

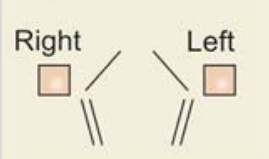

Right Left

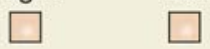

All small copies of basic scan: Basic views of BPD, HC, AC and $\mathrm{FL}$; Extended views to add face profile

Appendix 3: Potential detection rates based on present screening strategy

\begin{tabular}{lc}
\hline $\begin{array}{l}\text { Fetal anomaly chance } \\
\text { of being seen }\end{array}$ & $\begin{array}{l}\text { Detection rate of } \\
\text { different fetal anomaly }\end{array}$ \\
\hline Spina bifida & $90 \%$ \\
Anencephaly & $99 \%$ \\
Hydrocephalus & $60 \%$ \\
Major congenital heart problems & $25 \%$ \\
Diaphragmatic hernia & $60 \%$ \\
Exomphalos/gastroschisis & $90 \%$ \\
Major kidney problems & $85 \%$ \\
Major limb abnormalities & $90 \%$ \\
Cerebral palsy spasticity: never seen & \\
Autism: never seen &
\end{tabular}

accuracy. W omen should receive written details about their scan result. All scans should be carefully documented and archived. A ccurate record keeping is needed too with the pregnancy outcome recorded with sufficient detail. Use of the computer-based record keeping with the use of software should be encouraged and preferred which also helps in checking the quality and the audit of the unit/consultant. Regular audit of pregnancy outcome should be checked. 
Appendix 4: Pooled estimates of detection rate (DR), false-positive rate (FPR) and positive and negative likelihood ratios (LR + and LR -) of sonographic markers for trisomy 21 and estimated likelihood ratio (LR) of individual isolated markers ${ }^{16}$

\begin{tabular}{|c|c|c|c|c|c|}
\hline Marker & DR $(95 \% \mathrm{CI})(\%)$ & FPR $(95 \% \mathrm{CI})(\%)$ & $\mathrm{LR}+(95 \% \mathrm{Cl})$ & LR - $(95 \% \mathrm{CI})$ & $\begin{array}{l}\text { LR isolated } \\
\text { marker* }\end{array}$ \\
\hline Intracardiac echogenic focus & $24.4(20.9-28.2)$ & $3.9(3.4-4.5)$ & $5.83(5.02-6.77)$ & $0.80(0.75-0.86)$ & 0.95 \\
\hline Ventriculomegaly & $7.5(4.2-12.9)$ & $0.2(0.1-0.4)$ & $27.52(13.61-55.68)$ & $0.94(0.91-0.98)$ & 3.81 \\
\hline Increased nuchal fold & $26.0(20.3-32.9)$ & $1.0(0.5-1.9)$ & $23.30(14.35-37.83)$ & $0.80(0.74-0.85)$ & 3.79 \\
\hline Echogenic bowel & $16.7(13.4-20.7)$ & $1.1(0.8-1.5)$ & $11.44(9.05-14.47)$ & $0.90(0.86-0.94)$ & 1.65 \\
\hline Mild hydronephrosis & $13.9(11.2-17.2)$ & $1.7(1.4-2.0)$ & $7.63(6.11-9.51)$ & $0.92(0.89-0.96)$ & 1.08 \\
\hline Short humerus & $30.3(17.1-47.9)$ & $4.6(2.8-7.4)$ & $4.81(3.49-6.62)$ & $0.74(0.63-0.88)$ & 0.78 \\
\hline Short femur & $27.7(19.3-38.1)$ & $6.4(4.7-8.8)$ & $3.72(2.79-4.97)$ & $0.80(0.73-0.88)$ & 0.61 \\
\hline ARSA & $30.7(17.8-47.4)$ & $1.5(1.0-2.1)$ & $21.48(11.48-40.19)$ & $0.71(0.57-0.88)$ & 3.94 \\
\hline Absent or hypoplastic NB & $59.8(48.9-69.9)$ & $2.8(1.9-4.0)$ & $23.27(14.23-38.06)$ & $0.46(0.36-0.58)$ & 6.58 \\
\hline
\end{tabular}

*Derived by multiplying the positive LR for the given marker by the negative LR of each of all other markers, except for short humerus. ARSA, aberrant right subclavian artery; NB, nasal bone

\section{GENETIC SONOGRAM}

The 'Genetic Sonogram' should be a part of 'anomaly scan'. The genetic sonogram will help in identifying the fetus at the risk of fetal aneuploidy. ${ }^{18-20}$ The genetic sonogram involves the evaluation of presence or absence of soft markers between 16 and 20 weeks (A ppendix 4). Soft markers are the obstetric ultrasound findings, which are considered variants of normal but are noteworthy because they also increase the risk for underlying fetal aneuploidy. ${ }^{5,6}$ Presence of soft markers may be associated with nonchromosomal malformations also. The presence of soft markers increases the risk for fetal aneuploidy but is not diagnostic. Individual soft markers will vary in the degree of association with fetal aneuploidy. It has become practice to estimate the degree of association as a $L R$ by which the a priori background risk is altered. Detection of multiple soft markers will increase the significance of the finding, compared with seeing the same marker in isolation. In addition, maternal serum testing screening tool can complement and enhance the overall screening process. Providing an accurate assessment of fetal genetic risk require the ability to integrate known factors before patients can make an informed choice about proceeding with invasive diagnostic testing. Guidance on screening for aneuploidy is included in A ppendix 4.

Recently, meta-analysis done by Nicolaides and group have suggested new soft markers (A RSA, hypoplastic/ absent nasal bone, mild ventriculomegaly with likely hood ratio) as well as changed the likely hood ratios of the markers (A ppendix 4). ${ }^{16}$

\section{Thickened Nuchal Fold-Likelyhood Ratio' ${ }^{16}: \mathbf{3 . 8}$}

1. A thickened nuchal fold significantly increases the risk of fetal aneupl oidy. Expert review is recommended, and karyotyping should be offered.

2. A thickened nuchal fold is associated with congenital heart disease and rarely with other genetic syndromes.

\section{Mild Ventriculomegaly-Likelyhood Ratio' ${ }^{16}: 3.81$}

1. Cerebral ventricles greater than or equal to $10 \mathrm{~mm}$ are associated with chromosomal and central nervous system pathology. Expert review should be initiated to obtain a detailed anatomic evaluation looking for additional malformations or soft markers, laboratory investigation for the presence of congenital infection or fetal aneuploidy. Fetal MRI as an additional imaging technique may be of help.

2. Neonatal assessment and follow-up are important to rule out associated abnormalities and are important because of the potential for subsequent abnormal neurodevelopment.

\section{Echogenic Intracardiac Focus Likelyhood Ratio' 1}

1. Echogenic intracardiac focus (ECF) should be evaluated and reported as part of the 4-chamber cardiac review.

2. Women with right-sided, biventricular, multiple, particularly conspicuous, or nonisolated ECF should be offer referral for expert review and possiblekaryotyping.

\section{Mild Pyelectasis Likelyhood Ratio' ${ }^{16}: 1$}

1. If pyelectasis is visualized, the renal pelvis should be measured in the anterior/posterior diameter.

2. All fetuses with renal pelvic measurements $>5 \mathrm{~mm}$ should have a neonatal ultrasound, and those having measurements $>10 \mathrm{~mm}$ should be considered for a regular follow-up scan.

3. Isolated mild pyelectasis does not require fetal karyotyping.

4. Referral for pyelectasis should be considered with additional ultrasound findings and (or) in women at increased risk for fetal aneuploidy owing to maternal age or maternal serum screen results. 
Screening for Birth Defects Strategies for Developing Low Resource Countries

\section{Single Umbilical Artery}

1. A ssessment of cord vessels is considered a part of the routine obstetric ultrasound at 18 to 20 w eeks.

2. The finding of a single umbilical artery (SU A) requires a more detailed review of fetal anatomy, including kidneys and fetal heart (fetal echo).

3. An isolated SUA does not warrant invasive testing for fetal aneuploidy.

\section{Echogenic Bowel Likelyhood Ratio ${ }^{16:} 1.65$}

1. Echogenic bowel should be identified by comparison with the echogenicity of surrounding bone using an appropriate transducer and gain setting. Bowel echogenicity equal to or greater than bone is significant.

2. Echogenic bowel is associated with both chromosomal and nonchromosomal abnormalities. Expert review is recommended to initiate the detailed ultrasound evaluation looking for additional structural anomalies or other soft markers of aneuploidy, detailed evaluation of the fetal abdomen looking for signs of bowel obstruction or perforation, detailed evaluation of placental characteristics. Detailed maternal work up for serum screening tests, evaluation for cystic fibrosis and infection should be done. The genetic counseling and fetal karyotype should be considered.

\section{Absent/Hypoplastic Nasal Bone ${ }^{16:}$ LR 6.58}

When Nasal bone is absent OR Hypoplastic (shorter by 1 / 11 of BPD ) chances of Trisomy 21 increase by 6.58 times in particular black women. In Indian population, $N$ asal bone is absent/hypoplastic in about $10 \%$ of genral population.

The incidence of an absent nasal bone is related to NT, $\mathrm{CRL}$ and ethnic origin as well as aneuploidy, being more common when the NT is high, the CRL is low and the mother is Black.

\section{Choroid Plexus Cysts}

1. Isolated choroid plexus cysts (CPCs) require no further investigation when maternal age or the serum screen equivalent is less than the risk of a 35-year-old.

2. Fetal karyotyping should only be offered if isolated CPCs are found in women 35 years or older or if the maternal serum screen is positive for either trisomy 18 or 21.

3. All women with fetal CPCs and additional malformation should be offered referral and karyotyping.

\section{Mega Cisterna Magna}

1. An isolated mega cisterna magna is not an indication for fetal karyotyping.
2. With a mega cisterna magna, expert review is recommended for follow-up ultrasound, fetal MRI and investigations.

3. If the mega cisterna magna is seen in association with other abnormal findings, fetal karyotyping should be offered.

\section{Short Femur Length and Short Humerus Length Likelyhood Ratio: ${ }^{16} 0.1$ and Likelyhood Ratio: ${ }^{16} 0.78$}

If a femur and/or humerus appear abnormal or measures short on screening ultrasound, other long-bones should be assessed and referral with follow-up ultrasound considered.

\section{PRENATAL DIAGNOSTIC TESTS}

Commonly practiced invasive prenatal diagnosis techniques are chorionic villus sampling (CVS), amniocentesis, less commonly cordocentesis or percutaneous umbilical blood sampling (PUBS), fetal tissue sampling. Invasive testing can be performed in the first trimester by CVS or in the second trimester by amniocentesis; and have been the two most common prenatal diagnostic procedures for decades. Both procedures are safe, with an equivalent $0.5 \%$ risk of procedure-induced pregnancy loss. ${ }^{17}$ W hen performed prior to the routine sampling window of 15 weeks, amniocentesis may increase the risk of talipes equinovarus, the highest risk being encountered prior to 13 weeks' gestation. When CVS is performed prior to 9 weeks' gestation, there may be an increased risk of limb reduction defects. ${ }^{17}$ There are wide variations in utilization, operator skills, quoted procedure risks, actual observed risks, and patient choices that come from highly variable counseling as to those risks. The laboratory analysis of both procedures is reliable. CVS has a 1 to $2 \%$ incidence of confined placental mosaicism, requiring additional evaluation in some cases. M ost studies comparing CVS to amniocentesis in skilled hands have found equivalency of risks. Cordocentesis has fewer indications, is performed in the late second trimester of pregnancy, but allows direct laboratory testing from fetal blood. Experienced operators should perform all invasive procedures under continuous ultrasound guidance. Patient counseling should include an evaluation of the procedural risk associated with each individual case with its background risk. In general, patients are allowed to resume most daily activities after the procedure in India. Formal informed consent for invasive procedure should be obtained before the procedure.

\section{Chorionic Villus Sampling}

CVS is a test where a small piece of chorion frondosum (placental tissue) is removed and used for genetic testing. 
CVS is the most common first trimester invasive prenatal diagnosis technique for evaluation of fetal karyotype, molecular and biochemical abnormalities. CVS should not be performed before 10 weeks gestation because of the risk of transverse limb reduction defects. ${ }^{13-15}$ CVS should be performed by an operator using concurrent ultrasound. The operator should have adequate training and should continue performing sufficient numbers annually to maintain expertise.

\section{Indications for CVS}

- An abnormal first trimester screening by USG with/ without serum biochemistry indicating increased risk for chromosome problems (screen positive).

- Finding of fetal abnormality on ultrasound a previous child with a chromosome abnormality.

- Parents carry a chromosome translocation (rearrangement) or evaluation for the single gene disorder like thalassemia, Tay-Sachs, sickle cell anemia and DM D/ $\mathrm{CAH} / \mathrm{CF}$, etc.

- Skin disorders: epidermolysis bullosa dystrophica, albinism, ichthyosis.

\section{Advantages of CVS}

A n early result is advantageous for the patient, in that, in cases of an unaffected pregnancy the anxiety is relieved and in cases of affected pregnancy early termination of pregnancy can be undertaken with lower complication rate and less emotional stress than when termination follows amniocentesis at a later gestational age.

\section{Disadvantages and Risks of CVS}

A. Confined placental mosaicism: A discrepancy between the chromosomes in the chorionic and fetal tissues, is a biologic placental factor, which is present in 1 to $2 \%$ of pregnancies

B. Maternal contamination: W ith decidual tissue

C. Pregnancy loss: In addition to the background risk of spontaneous pregnancy loss in the advanced maternal age group, the procedure related loss is about 1 to $2 \%$ in comparison to the 0.5 to $1 \%$ risk for amniocentesis. ${ }^{12}$

D. Limb or facial anomalies: The risk of limb or facial anomalies is higher if CVS is done at a gestational age earlier than 9 weeks, hence universally, CV S is generally restricted to greater than or equal to 10 weeks. These anomalies may be due to a vascular disruption sequence event, which may be associated with the CV S procedure.

\section{Amniocentesis}

\section{Indications}

A mniocentesis ${ }^{22}$ is usually performed for determination of fetal karyotype, molecular and biochemical abnormalities.
The most common test performed on the amniotic fluid is the fetal karyotype from fetal and membrane cells in the amniotic fluid after tissue culturing or fetal chromosomal evaluation by direct fluorescent in situ hybridization (FISH) techniques. A mniocentesis should be performed with concurrent ultrasound should be used. A mniocentesis is usually performed from 15 weeks gestation and should not routinely be performed before 14 weeks gestation because of the increased risk of adverse outcome.

Some of the most common indications for amniocentesis are:

- For chromosomal analysis in the fetus who is screen positive after USG and/or serum biochemistry

- A previous child with a chromosome abnormality or metabolic disorder

- One or both parents carry a chromosome translocation (rearrangement) or

- Both parents carriers of a genetic disease such as thalassemia minor, Tay-Sachs, sickle cell anemia, etc.

- Finding of a fetal abnormality on ultrasound suggestive of chromosomal anomaly

- Risk of fetal infection

- Sex determinations (only for X-linked disease, CAH, DMD)

- Biochemical disorders and inborn errors of metabolism screening in fetus

- Study of microdeletions in fetus.

\section{Risks of Amniocentesis}

\section{Fetal Loss}

Fetal loss after amniocentesis is 0.5 to $1 \%$ above the background loss. ${ }^{14,21}$

\section{Infection}

The risk of infection introduced at the time of the amniocentesis is estimated to be 1 to 2 in 3,000 procedures. ${ }^{4}$

\section{Fetal Injury}

Serious fetal injuries at the time of amniocentesis are rare with continuous ultrasound guidance.

\section{Other Complications}

Include leakage of amniotic fluid, bleeding and uterine irritability.

These complications are estimated to occur in $1 \%$ of procedures and are generally self-limited.

Comparing various approaches of prenatal diagnostic techniques:

A. Transabdominal CVS vs second trimester amniocentesis: A subgroup of Denmark compared transabdominal CV S 
with second trimester amniocentesis and found no significant difference in the total pregnancy loss between the two procedures ( 6.3 vs $7 \%$; R R: $0.90 ; 95 \% \mathrm{Cl}: 0.66$ 1.23).

B. Transabdominal vs transcervical CVS: Compared with transabdominal CVS, total pregnancy loss and spontaneous miscarriages were higher after transcervical CVS. Vaginal bleeding following the procedure was much more common after transcervical CVS, although there was no difference in the incidence of vaginal bleeding later in pregnancy. There was no significant difference in the amniotic fluid leakage following the procedure and prelabor spontaneous rupture of membranes before 28 weeks.

C. Early amniocentesis (EA) vs transabdominal CVS: Spontaneous miscarriages after early amniocentesis are more common (RR: 1.76; $95 \% \mathrm{Cl}: 1.17-2.64$ ).

\section{Cordocentesis}

Fetal blood sampling provides information that is not obtainable by other techniques for fetal assessment. It has tremendous fetal diagnostic and therapeutic applications, and exciting research potential. It allows the direct estimation of fetal hemoglobin, hematocrit, blood group, platelet count, reticulocyte, and white blood cell count for prenatal diagnosis of fetal anemia, thrombocytopenia, etc. Cord blood gives a better and quicker chromosomal preparation than with chorionic villi or amniotic fluid. Congenital infections can be diagnosed by serology, direct identification of the viral particles by electron microscopy of fetal blood, cultures of fetal blood, and indirect parameters like platelet count, total leukocyte count, differential count, and liver enzymes are carried out to arrive at a diagnosis. Fetal blood sampling (direct ultrasound guided fetal blood sampling) should also be performed or closely supervised by operators trained in this procedure who perform a sufficient number of such samplings to ensure technical success (i.e. sampling fetal blood), and to minimize the complication rate.

\section{Indications}

- Rh immunization-Hb, blood group, intrauterine transfusion

- Rapid fetal Karyotype (late pregnancy)

- Hematology-Hb, Factor VIII, IX deficiency, platelets

- Congenital infections-PCR, IgM (TORCH), parvovirus

- All indications similar to amniocentesis.

Indications are decreasing as prenatal diagnosis of these conditions can now be done by CVS or by amniocentesis.
The procedure-related fetal loss rate for cord blood sampling is 1 to $2.6 \%$. The overall mortality (including background morbid condition of a diseased fetus) appears to be around 5.0\% (between 3.84 and 5.87\%), but fetal loss rate directly related to the procedure seems to be around $1 \%$ (between 0.88 and $0.98 \%$ ) only. Fetal loss rate is closely related to the state of the fetus and indication of the procedure.

Transient bradycardia varying from 15 to 134 seconds may be seen in 3 to $9 \%$. Complications and success in obtaining the blood sample depends on the experience of the operator.

\section{Rhesus Status}

Rhesus status should be available or obtained in every case before the prenatal invasive diagnosis. A nti-D I g should be given to all nonsensitized RhD-negative women with $\mathrm{Rh}$ positive husband after the invasive prenatal diagnosis like amniocentesis, chorion villus sampling, fetal blood sampling and other intrauterine procedures, e.g. insertion of shunts, embryo reduction. A dose of $50 \mathrm{mcg}$ is recommended for prophylaxis following sensitizing events up to 20 weeks of pregnancy and for all events after 20 weeks, at least $100 \mathrm{mcg}$ anti-D Ig should be given followed by a test to identify fetomaternal hemorrhage. Final dose has to be calculated after the quantification of fetomaternal hemorrhage. fetomaternal hemorrhage greater than $4 \mathrm{ml}$ red cells of fetal blood, additional anti-D I $g$ should be given as per-requirement.

\section{CONCLUSION}

The choice between first trimester combined testing (NT with maternal serum screening), integrated screening (NT, first and second trimester maternal serum screening), CV S, amniocentesis, second trimester serum screening should be based on of informed consent. This should take into consideration the risks of the test, timing, method of termination, which may be considered (if affected), and accuracy of the test. All women should be offered first trimester (11 to $13+6$ weeks) and a mid trimester (18-20 weeks) ultrasound scans with or without serum biochemistry screening. A mniocentesis and CVS are very useful techniques for fetal care, quite safe obstetric procedures in expert hands, with backup requirement of a good genetic laboratory. They require skill, and should preferably be done in referral centers to maximize safety, and optimize patient management. In addition, there is need for improved and more specific noninvasive screening methods to identify 
women whose fetuses are at risk of congenital or genetic disease, to minimize number of women requiring PND procedures. Obstetricians play a key role in prenatal diagnostic and genetic services, by screening, counseling and timely referrals.

\section{GENERAL PRINCIPLES FOR PRENATAL DIAGNOSIS PROGRAMS}

1. All patients considering prenatal diagnosis should have access to professionals who are knowledgeable in the field appropriately minimally trained for doing the procedures. The prenatal diagnostic service units should use state of the art ultrasound equipment. Each specialized prenatal diagnostic service requires the services of a multidisciplinary team of a specialist in obstetric ultrasound, clinical geneticist, genetic counsellor, obstetrician (with specializing in prenatal diagnosis and management of fetal abnormality preferred), pediatrician, pediatric surgeon and laboratory. There should be at least one specialized prenatal diagnostic service center for all states of India.

2. A suggested minimum caseload of 50 invasive procedures per year is recommended per practitioner and 100 prenatal specimens for the genetic laboratory in order to maintain an appropriate level of competence. Exceptions to this minimum caseload may be justified because of unique geographic circumstances.

3. Each patient should have an appropriate assessment of family history and genetic counseling prior to undergoing invasive prenatal diagnosis.

4. Counseling should be given in a nondirective manner in order to allow an informed choice by the couple.

5. The distinction between screening and diagnostic investigations should be clarified, including the frequency of abnormal results, false-positive and falsenegative tests. A ccuracy of results, frequency of need for repeats testing, and risks of pregnancy loss are of particular relevance with invasive prenatal diagnosis procedures. The couple should be reminded that normal test results do not rule out every genetic or structural abnormality in their fetus.

6. Prior to embarking on prenatal diagnosis testing, couples should be made aware of the full range of options when confronted with an abnormal test result. Prior commitment to termination of pregnancy following the diagnosis of fetal abnormality is not a prerequisite for prenatal diagnosis. Each center must be aware of the local, regional, national, and international policies and protocols related to termination of pregnancy, and advise the couple of such before undertaking prenatal diagnosis. This is particularly important at gestations beyond 20 weeks.
7. Determination of fetal sex for the purpose of sex selection procedures on a nonmedical basis is inappropriate and against the law in India.

8. When a fetal anomaly is found, a multidisciplinary group should be involved for the management of the patient and the fetus.

\section{REFERENCES}

1. Royal College of Obstetricians and Gynaecologists (Great B ritain). U Itrasound screening for fetal abnormalities. Report of the RCOG working party. London: Royal College of Obstetricians and Gynaecologists Press; 1997. p 1-58.

2. N eilson JP. U Itrasound for fetal assessment in early pregnancy. Cochrane Review. The Cochrane L ibrary I ssue 3. Oxford: J ohn W iley and Sons, Ltd; 1999.

3. W ald N J, Cuckle HS, Densem JW, Kennard A, Smith D. $M$ aternal serum screening for Down's syndrome: The effects of routine ultrasound scan determination of gestational age and adjustment for maternal weight. Br J Obstet Gynaecol 1992; 99(2):144-149.

4. B oyd PA, Chamberlain P, Hicks NR. 6-year experience of prenatal diagnosis in an unselected population in Oxford, UK. L ancet 1998;352(9140):1577-1581.

5. Whittle MJ. Ultrasonographic 'soft markers' of fetal chromosomal defects. BMJ 1997;314(7085):918.

6. Chodirker B M , Cadrin C, Davies GAL, Summers A M , W ilson RD, Winsor EJT, Y oung D. Canadian guidelines for prenatal diagnosis: genetic indications for prenatal diagnosis. J Soc Obstet Gynaecol 2001 J un;23(6):525-531.

7. Snijders RJM, Sundberg K, Holzgreve W, Henry G, N icolaides $\mathrm{KH}$. M aternal age- and gestation-specific risk for trisomy 21. Ultrasound Obstet Gynecol 1999 M ar;13(3):167-170.

8. Fetal Medicine Foundation. Available from: www.fetal medicine.com.

9. K rantz DA, Larsen JW , Buchanan PD, M acri J N. First trimester Down syndrome screening: free beta-human chorionic gonadotropin and pregnancy-associated plasma protein A. A m J Obstet Gynaecol 1996 Feb;174(2):612-616.

10. Prashant $A, A$ shini $A$. Evaluation of applicability of standard growth curves to Indian women by fetal biometry. J South A sian Federation O bstet Gynecol 2009 Sep-Dec;1(3):55-61.

11. Snijders RJ, Noble P, Sebire N, Souka A, Nicolaides KH. UK multicentre project on assessment of risk of trisomy 21 by maternal age and fetal nuchal-translucency thickness at 10-14 weeks of gestation. L ancet 1998 A ug;352(9125):343-346.

12. A nandakumar $C$, W ong $Y C$, A nnapoorna $V$, A rulkumaran $S$, Chia D, Bongso A, Ratnam SS. A mniocentesis and its complications. A ust NZJ Obstet JOGC Gynaecol 1992 M ay;32(2):97-99.

13. Sundberg K, B ang J, Smidt-J ensen S, B rocks V , L undsteen C, Parner J, K eiding N, Philip J. Randomized study of risk of fetal loss related to early amniocentesis versus chorionic villus sampling. L ancet 1997;350(9079):697-703.

14. Medical Research Council European trial of chorion villus sampling. M RC working party on the evaluation of chorion villus sampling. Lancet 1994 J un;337(8756):1491-1499.

15. Evaluation of chorionic villus sampling safety. WHO/PA HO Consultation on CV S. Prenat Diagn 1999 Feb;19(2):97-99. 
16. A gathokleous $M$, Chaveeva $P$, Poon LCY, Kosinski $P$, $\mathrm{Nicolaides} \mathrm{KH}$. M eta-analysis of second-trimester markers for trisomy 21. Ultrasound Obstet Gynecol 2013;41:247-261. Published online 24 J anuary 2013 in Wiley Online Library (wileyonlinelibrary.com).

17. A mniocentesis and chorionic villus sampling (Green top 8). Royal College of Obstetrics and Gynecology; J une 2010.

18. DeV ore GR, Romero R. Combined use of genetic sonography and maternal serum triple marker screening: an effective method for increasing the detection of trisomy 21 in women younger than 35 years. J Ultrasound M ed 2001 J un;20(6):645-654.

19. Benn PA, K aminsky LM, Y ing J, Borgida A F, Egan JF. Combined second-trimester biochemical and ultrasound screening for Down syndrome. Obstet Gynecol 2002 Dec;100(6):1168-1176.

20. Hobbins J C, L ezotte DC, Persutte W H, DeV ore GR, B enacerraf $B R$, Nyberg DA , V intzileos A M , Platt L D, C arl son DE, B ahadoSingh RO, et al. A n 8-center study to evaluate the utility of mid-term genetic sonograms among high-risk pregnancies. J Ultrasound M ed 2003 J an;22(1):33-38.

21. Radhakrishnan P. Effectiveness of the First trimester scan in detection of major structural anomalies. Rhodes, Greece: Presented at the 9 th World Congress in Fetal M edicine.

22. Alfirevic Z, Sundberg K, Brigham S. A mniocentesis and chorionic villus sampling for prenatal diagnosis. Cochrane Database Syst Rev 2003;(3):CD 003252.

23. Syngelaki A, Chelemen T, Dagklis T, Allan L, Nicolaides KH. Challenges in the diagnosis of fetal nonchromosomal abnormalities at 11-13 weeks. Prenat Diagn 2011 Jan;31(1):90-102.

24. Whitlow BJ, Chatzipapas IK, Lazanakis M L, Kadir RA, Economides $D L$. The value of sonography in early pregnancy for the detection of fetal abnormalities in an unselected population B J O bstet Gynaecol 1999 Sep;106(9):929-936.

25. Vavilala S, Geeta K. The 11-13+6 weeks scan: where do we stand? A 5-year review at Fernandez Hospital. Int J Infertility Fetal M ed 2011 A ug;2(2):65-69.

\section{ABOUT THE AUTHORS}

\section{Prashant Acharya}

Consultant, Department of Fetal M edicine and High Risk Obstetric Care, Paras A dvanced C enter for Fetal M edicine, A hmedabad, Gujarat; Vice President, FOGSI, 2012; Ex. Chairperson, Genetic and Fetal M edicine Committee, FOGSI 2009-2012; Coordinator, IIS, India 2012 (ISU OG International Symposium)
Correspondence Address: Paras-A dvanced Center for F etal Medicine, 2/B, Virvijay Society, Opp Sanghvi High School Vijaynagar Road, Naranpura, A hmedabad-380013, Gujarat, India e-mail: ashinprashant@hotmail.com, ashiniprashant@gmail.com

\section{Jaideep Malhotra}

President Elect, ASPIRE 2014; Infertility and ART Specialist; Consultant, Department of Obstetrics and Gynecology; Director M edical Services, Rainbow Hospital, A gra, U ttar Pradesh I ndia; V ice President, FOGSI 2010

\section{Narendra Malhotra}

Professor, Dubrovnik International University; President, FOGSI 2008; Consultant, Infertility Specialist, D epartment of Obstetrics and Gynecology; M anaging Director, Rainbow Hospital, A gra Uttar Pradesh, India

\section{S Suresh}

Consultant, M ediscan Center, Chennai, Tamil Nadu, India

\section{Ashok Khurana}

Consultant Radiologist, Department of Radiology, New Delhi, India

\section{Chander Lulla}

Consultant Radiologist, Ria Clinic, M umbai, M aharashtra, India

\section{Hriday Acharya}

Intern, Civil Hospital, A hmedabad, Gujarat, India

\section{Neharika Malhotra Bora}

Lecturer, Department of Obstetrics and Gynecology, B harati Vidyapeeth M edical College, Pune, M aharashtra, India

\section{Rishabh Bora}

2nd $Y$ ear Resident, Department of Radiology, B harati Vidyapeeth M edical College, Pune, M aharashtra, India

\section{Keshav Malhotra}

Intern, D istrict H ospital, A gra, U ttar Pradesh, India 